\title{
Space Radiation of Solar Storm: A Meeting Report in Taiwan
}

\author{
Jyh-Woei Lin
}

\section{ABSTRACT}

Solar storm was an effect when Sun was active. Solar flares flame released a large amount of energy and caused a large-scale explosion. A large amount of coronal matter was ejected into space by plasma composed of electrons and protons. Their shock waves or magnetic clouds and the earth Magnetic storms generated by the interaction of magnetic fields caused disturbances and squeezing of the earth's magnetosphere. A solar flare was a phenomenon of solar storm. It had huge eruptions of electromagnetic radiation. The sudden electromagnetic energy traveled with the speed of light. Large solar flare might affect the effects of reliability of electronic components in satellites and could cause economic losses by soft error and could affect human health through the space radiation, especially causing cancer.

Keywords: Cancer, Earth's magnetosphere, Electronic Components, Soft Error, Solar flares, Solar storm, Space Radiation.
Published Online: November 11, 2021

ISSN: $2684-446 \mathrm{X}$

DOI : $10.24018 /$ ejgeo.2021.2.6.202

\section{Jyh-Woei Lin*}

Binjiang College, Nanjing University of Information Science \& Technology, China.

Department of Electrical Engineering, Southern Taiwan University of Science and

Technology, Tainan, Taiwan.

(e-mail: pgjw11966@gmail.com)

*Corresponding Author

\section{INTRODUCTION}

Solar storm was defined as when the sun was active, the powerful Solar flares flame instantly released a large amount of energy and caused a large-scale explosion, accompanied by a large amount of coronal matter, which was ejected into space by plasma composed of electrons and protons; solar wind shock waves or magnetic clouds and the earth Magnetic storms generated by the interaction of magnetic fields caused disturbances and squeezing of the earth's magnetosphere. According to Faraday's law of electromagnetic induction has been changed in magnetic fields, which generated electromotive force and caused electronic devices to generate induced currents [1]-[4].

\section{DISCUSSION}

A solar flare (Fig. 1) was a very important phenomenon of solar storm, which had huge eruptions of electromagnetic radiation. The sudden electromagnetic energy traveled with the speed of light. Therefore, any effect upon the sunlit side of Earth's exposed outer atmosphere occurred and could be observed. If the ejection of solar flare was along the direction of the Earth, particles associated with this disturbance penetrated into the upper atmosphere incl. ionosphere and caused bright auroras (Fig. 2) and ionospheric anomaly e.g., the precursors of large earthquake [5]. This might also disrupt long range radio communication. When ejecting to reach Earth, usually, it has taken few days for the solar plasma. Large solar flare might affect the effects of reliability of electronic components in satellites and could cause economic losses through the space radiation by soft error [6]-[8]. The space radiation also could affect human health, especially causing cancer (Particle Physics and Beam Delivery Core Laboratory, Taiwan).

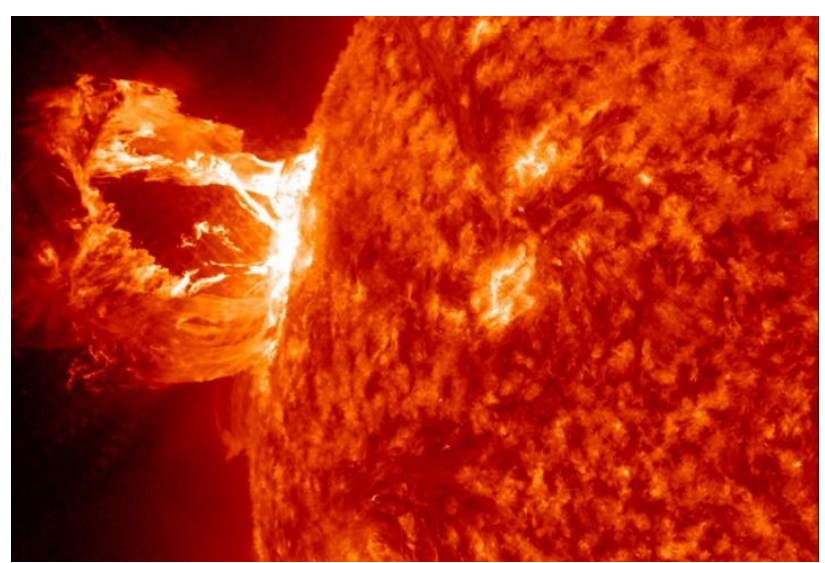

Fig. 1. A solar flare on June 9, 2017 (UTC) (National Aeronautics and Space Administration, NASA).

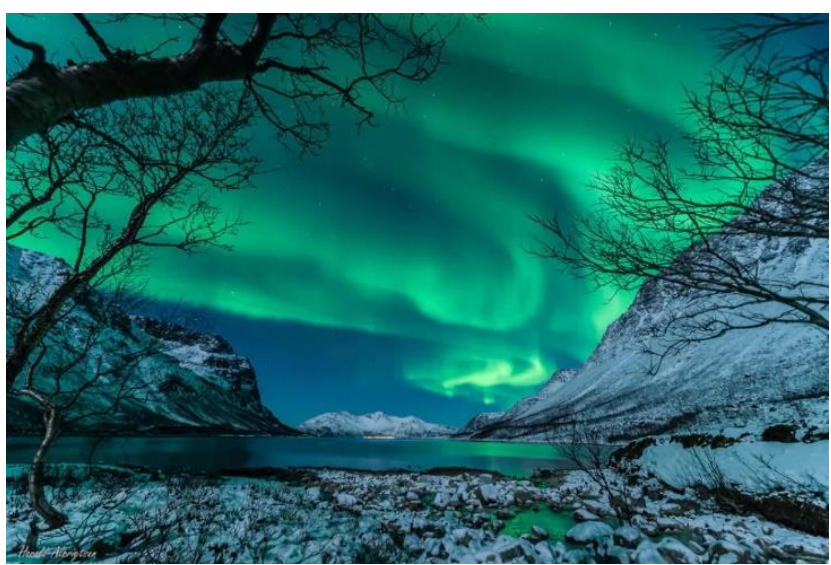

Fig. 2. An aurora on Jan. 9, 2014 (UTC) in Norway (NASA). 


\section{CONCLUSION}

Solar storm was defined as when the sun was active, the powerful Solar flares flame instantly released a large amount of energy and caused a large-scale explosion, accompanied by a large amount of coronal matter, which was ejected into space by plasma composed of electrons and protons; solar wind shock waves or magnetic clouds and the earth Magnetic storms generated by the interaction of magnetic fields caused disturbances and squeezing of the earth's magnetosphere. A solar flare was a phenomenon of solar storm, which had huge eruptions of electromagnetic radiation. The sudden electromagnetic energy traveled with the speed of light. Therefore, any effect upon the sunlit side of Earth's exposed outer atmosphere occurred and could be observed. Large solar flare might affect the effects of reliability of electronic components in satellites and could cause economic losses by soft error and human health, especially causing cancer, through the space radiation.

\section{ACKNOWLEDGMENTS}

The author is also grateful the supporting of National Aeronautics and Space Administration, NASA USA and Particle Physics and Beam Delivery Core Laboratory, Taiwan (For the meeting "Space Radiation" on 20 October 2021 in Beam Delivery Core Laboratory, Taiwan). The author is also grateful the supporting of Prof. Dr Yuan Mei in Taiwan and all of my friends in Taiwan.

\section{CONFLICTS OF INTEREST}

The author declares that there is no conflict of interest.

\section{AUTHOR CONTRIBUTIONS}

designed the research; performed the research; analyzed the data; and wrote the paper.

\section{REFERENCES}

[1] Borovsky JE, Denton MH. Magnetic field at geosynchronous orbit during high-speed stream-driven storms: Connections to the solar wind, the plasma sheet, and the outer electron radiation belt. Journal of Geophysical Research: Space Physics, 2010;115:A8. doi: doi.org/10.1029/2009JA015116.

[2] Dremukhina LA, Lodkina LG, Yermolaey YI. Relationship between the Parameters of Various Solar Wind Types and Geomagnetic Activity Indices. Cosmic Research. 2018;56:426-433. doi: 10.1134/S0010952518060011

[3] Kalegaev VV, Vlasova NA, Nazarkov IS, Melkova SA. Magnetospheric access for solar protons during the January 2005 SEP event. Journal of Space Weather and Space Climate. 2018;8:A55. doi:10.1051/swsc/2018040.

[4] Battersby S. Core Concept: What are the chances of a hazardous solar superflare? Proceedings of the National Academy of Sciences of the United States of America (PNAS). 20191;116(47):23368-23370. doi: 10.1073/pnas.1917356116.

[5] Lin JW, Chiou JS. Detecting Total Electron Content Precursors Before Earthquakes by Examining Total Electron Content Images Based on Butterworth Filter in Convolutional Neural Networks, IEEE Access. 2020;8:10478-110494. doi: 10.1109/ACCESS.2020.3001337.

[6] Slayman C. Soft error trends and mitigation techniques in memory devices, IEEE 2011 Proceedings - Annual Reliability and Maintainability Symposium, 1197-1122, 2011. doi: 10.1109/RAMS.2011.5754515.
[7] Lin JW. A Solar Flare on 10 September 2014 was Associated with Two F/A-18C Hornets Crashing in the Western Pacific Ocean. Journal of Geography, Environment and Earth Science International. 2015;2(3):158-162. doi: 10.9734/JGEESI/2015/16620.

[8] Eastwood JP, Biffis E, Hapgood MA, Green L, Bisi MM, Bentley RD, Wicks R, McKinnell LA, Gibbs M, Burnett C. The Economic Impact of Space Weather: Where Do We Stand? Risk Analysis. 2017;37(2):206-218. doi:10.1111/risa.12765.

[9] Ferguson DC, Worden SP, Hastings DE. The Space Weather Threat to Situational Awareness, Communications, and Positioning Systems, IEEE Transactions on Plasma Science, 2015;43(9):3086-3098. doi: 10.1109/TPS.2015.2412775.

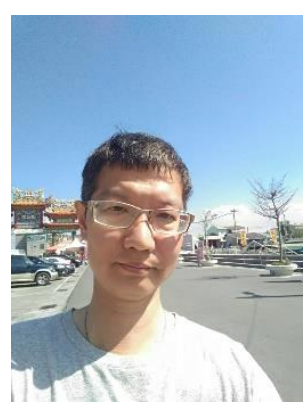

Jyh-Woei Lin received the B.Sc. degree from the Department of Physics, Chung Yuan Christian University, Chung Li City, Taiwan, in 1989, the M.Sc. degree from the Institute of Geophysics, National Central University, Chung Li City, in 1991, the Ph.D. degree from the Institut für Geophysik, ClausthalZellerfeld, Technische Universit ät Clausthal, Germany, in 2000, and the Ph.D. degree from the Department of Electrical Engineering, Southern Taiwan University of Science and Technology, Tainan, Taiwan, in 2019. Since 2019, he is a researcher in the Department of Electrical Engineering, Southern Taiwan University of Science and Technology. Until 2021, he has 71 SCI (SCIE) and EI papers include artificial intelligence, space physics, geophysics, medical sciences, and remote sensing; especially four books' publications in Germany and two books in USA. After September 2020, he is also as a Professor in Binjiang College, Nanjing University of Information Science \& Technology, Wuxi, Jiangsu Province, China. 